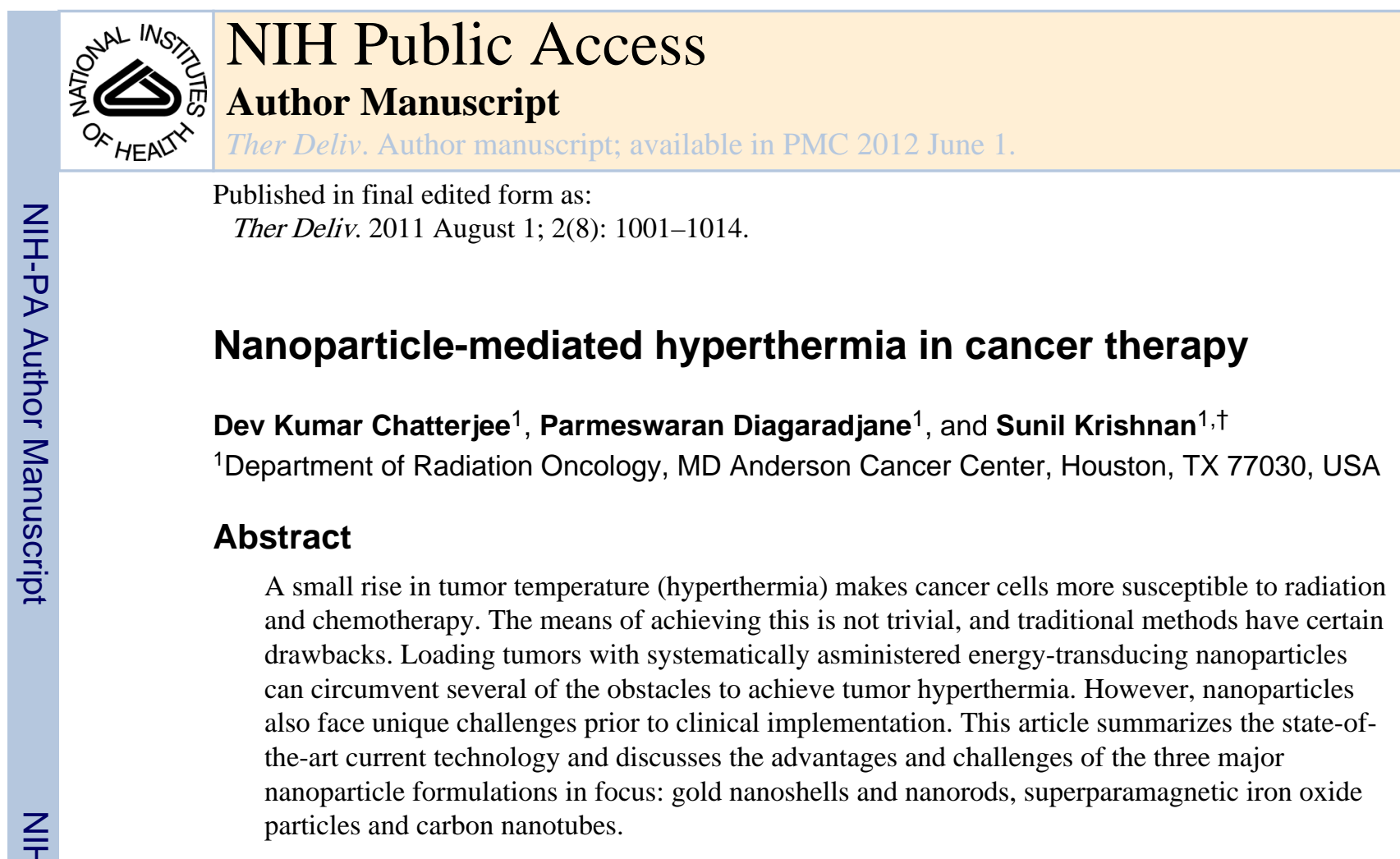

\title{
Hyperthermia in cancer therapy
}

\section{Clinical rationale \& biological basis for hyperthermia}

\begin{abstract}
Hyperthermia has a long history in the annals of cancer management. A correlation between erysipelas (a streptococcal skin infection) and tumor regression had been observed for over a century before William Coley first documented evidence of a relationship between infection and cancer regression in sarcoma patients in 1891 [1]. His attempts to recreate this phenomenon for the treatment of cancers culminated in the generation of cocktails of bacteria (Coley's toxin) that intentionally induced a fever to effect an antitumor response. While this probably represents among the first instances of the clinical use of hyperthermia for cancer therapy, it was also among the first demonstrations of the efficacy of immunotherapy. Since then, more localized and relatively safer methods of hyperthermia, either singly or in combination with conventional therapy, have been employed by many investigators to treat cancer (Box 1) [2-11].
\end{abstract}

\section{Box 1}

\section{Definitions}

- Hyperthermia, in a medical setting, is a rise in temperature of body tissues, globally or locally. In the field of cancer therapy where this elevation in temperature is induced intentionally, this definition can be further qualified based on the desired effect of this temperature rise on the tissue. If the temperature is raised high enough to cause immediate cellular death, largely

\footnotetext{
(C) 2011 Future Science Ltd

${ }^{\dagger}$ Author for correspondence: Tel.: +1 713563 2377, Fax: +1 713563 2366, skrishnan@mdanderson.org.

For reprint orders, please contact reprints@ future-science.com
}

Financial \& competing interests disclosure

This work was funded in part by NIH grants KL2 RR024149 05, 1R21CA133691-01, 1R01CA132032 and U01CA151886 to Sunil Krishnan. The authors have no other relevant affiliations or financial involvement with any organization or entity with a financial interest in or financial conflict with the subject matter or materials discussed in the manuscript apart from those disclosed. No writing assistance was utilized in the production of this manuscript. 
through necrosis mediated by irreparable coagulation of proteins and other biological macromolecules, the more accurate term to use is thermoablation. In contrast to thermoablation, hyperthermia refers to smaller temperature rises, usually to $40-45^{\circ} \mathrm{C}$, initiating a series of subcellular events, rendering the cells susceptible to various forms of damage including apoptosis, leading to subsequent cell death [70,71]. Other effects associated with hyperthermia include activation of immunological responses, enhancement of tumor blood flow and oxygenation via greater vascular perfusion and permeability, and a shift toward anaerobic metabolism resulting in decreased oxygen consumption and increased tissue oxygenation, all leading to an altered extracellular microenvironment.

- Nanoparticle is an umbrella term encompassing a variety of materials all sharing the common feature of having the longest dimension less than $100 \mathrm{~nm}$. This limitation is generally relaxed in the scientific parlance, with particles not greatly exceeding $100 \mathrm{~nm}$ also earning the sobriquet 'nano' (Figure 1). Nanoparticles can be solid, hollow or branching, and made from a very diverse selection of materials. The development of means to control and characterize at the nanoscale allows a degree of design unsurpassed by single-molecule entities. In the field of cancer therapy, nanoparticles afford many advantages over conventional therapeutic methods and are an ongoing research focus on a global scale.

Heat as a curative modality (thermoablation) has been explored in cancer by many researchers. The challenge facing thermoablation therapies mirrors that of surgery: the destruction of the tumor needs to be as complete as possible, while sparing normal tissues. Thermoablation became a topic of high interest with the advent of electromagnetic heating. However, despite demonstrations of delayed tumor regrowth following thermoablation, in general, the therapy falls short in curative prowess owing to the difficulties in precise control of temperature rise in different regions of the tumor resulting in residual cancerous tissue and tumor recurrence. By contrast, sub-ablative heating has a bigger therapeutic window since cell destruction is not desired. Mild temperature rise throughout the tumor may be accompanied by some heating of surrounding normal tissue, but since non-malignant cells have unimpaired heat-shock protection mechanisms, the toxicity profile for mild hyperthermia is usually very good.

The increasing interest in hyperthermia has been paralleled by an increasing understanding of the mechanism of action of this treatment modality. It is now known that tumor cells, tumor vascular endothelial cells, and normal cells are not inherently different in their sensitivity to heat-induced cytotoxicity. However, owing to inefficient blood flow and oxygen transport through the newly formed immature blood vessels within tumors, tumor cells reside in an acidotic and nutrient-deprived milieu that confers them with greater thermosensitivity [12]. This greater sensitivity of hypoxic areas to heat provides a compelling rationale for the combination of hyperthermia with radiation therapy since poorly perfused tumor cores are generally resistant to the effects of ionizing radiation, which depends on the generation of toxic oxygen radicals in well-perfused regions. The complementary effects of radiation and hyperthermia is further accentuated by the fact that tumor cells in the $\mathrm{S}$ phase of the cell cycle are relatively radioresistant. However, it is precisely in this phase that tumor cells are most sensitive to the effect of heat and become radio-sensitized. Thus hyperthermia acts as a potent and selective radiosensitizer by affecting those cancer cells that are naturally radioresistant. 
Hyperthermia can also be used to chemosensitize cancer cells. The degree of chemosensitization varies with the type and concentration of drug, the tumor type, the increase in tumor temperature and the time differential between the delivery of heat and chemotherapy. However, prior drug resistance does not appear to be a factor in the sensitivity to heat. Chemosensitization is highest when heat and chemotherapy are administered simultaneously, the effect diminishing at greater intervals between delivery of the two therapies [13]. In general, enhanced cytotoxicity of many chemotherapeutic agents increases in the range of $40.5-43^{\circ} \mathrm{C}$ [14]. However, clinically, temperatures above $41^{\circ} \mathrm{C}$ have been associated with higher toxicity and a low benefit:risk ratio. In general, hyperthermia with milder temperatures increases the destruction of cancer cells with chemotherapy, but not that of normal cells (i.e., chemotherapeutic side effects are minimized). However, there is a potentially wide variation in the thermal enhancement of a given agent among the different types of tumors. Also, different agents have different enhancement ratios for the same degree of temperature elevation in the same tumor type, indicating that the drug of choice at physiological temperatures may not be the drug of choice at elevated temperatures [14]. In general, however, even for drugs with good thermal enhancement for a particular tumor type, the enhancement of tumor toxicity due to heat is only seen at higher drug concentrations in the tumor. Hence, isolated perfusion of tumors with heated solutions of chemotherapeutics, providing a higher tumor drug concentration demonstrates a better response than systemic administration of the same drug. Several mechanisms have been proposed for this observed effect. Temperatures bordering on $42-$ $43^{\circ} \mathrm{C}$ have some cancer-specific cytotoxicity resulting from the impaired mechanisms of cancer cells to handle heat shock. Prolonged $(>1 \mathrm{~h})$ heating at lower temperatures increases tumor perfusion, and this results in greater delivery of chemotherapeutics especially to the poorly vascularized core. The lack of enhanced drug toxicity with loco-regional hyperthermia with potential improvement in response to advanced disease suggests that thermochemotherapy is a viable and important alternative to drug treatment alone.

Unfortunately, it is not often possible to elevate tumor temperature uniformly to the 42$43^{\circ} \mathrm{C}$ required for direct cytotoxicity. Fortuitously, it is fairly well established now that clinically achievable elevations of tumor temperature to approximately $40-42^{\circ} \mathrm{C}$ (mild temperature hyperthermia), although less effective as monotherapy against cancer, has potent efficacy as an adjunct to radiation therapy. This is largely driven by an increase in blood flow (often sustained for 1-2 days) [15] and oxygen delivery and a decrease in oxygen demand (due to hyperthermia-induced cell death and metabolic suppression) that converge to increase tumor tissue oxygenation. Increased perfusion also directly translates to increased delivery of chemotherapeutic drugs to the poorly vascularized tumor cores [16]. Such a complementary role for hyperthermia has been demonstrated in numerous clinical and preclinical studies of hyperthermia combined with chemotherapy or radiation therapy, with significant improvements in outcome demonstrated for tumors of the prostate, breast, bladder, brain, cervix, head and neck, lung, rectum and esophagus, among others. Typically, adding hyperthermia to the treatment regimen has not resulted in increased toxicity (especially for radiation therapy), but has contributed to better control, cure and/or palliation [17-24].

\section{Hyperthermia techniques}

While the rationale for adding hyperthermia to clinical regimens is compelling, the means to do so are not as straightforward. Three types of hyperthermia are traditionally employed in clinical practice - whole-body, regional and local hyperthermia (Figure 1). Whole-body hyperthermia is achieved by such methods as hot water blankets and thermal chambers. In theory, it should be used for metastatic cancer where focal hyperthermia would be ineffective. Regional hyperthermia depends on perfusing with heated liquids - the two 
popular techniques are perfusing the peritoneum with a heated solution of anticancer drugs for peritoneal cancers such as mesothelioma, and the perfusion of a part of the patient's blood, taken out and warmed ex vivo, into an artery supplying the limb containing the tumor $[25,26]$. Both whole-body and regional hyperthermia in general result in poor tumor specificity of treatment. While the toxic effects of hyperthermia are usually not severe, whole-body hyperthermia often causes gastrointestinal symptoms, such as diarrhea, nausea and vomiting, and may occasionally have serious cardiovascular side effects, such as myocardial ischemia, thrombosis and cardiac failure. Regional hyperthermia is invasive and involves significant challenges in set-up.

Local hyperthermia, in theory, has the advantage of being tumor focused. The three major methods of generation of local hyperthermia, in order of increasing invasiveness, are external, luminal and interstitial. Luminal hyperthermia uses special probes placed as close to the tumor as possible, such as in the lumen of the rectum for treatment of rectal cancers, whereas interstitial hyperthermia uses a grid of applicators/probes placed into tumor parenchyma for more uniform tumor heating. Heat sources can be inserted into the probes and applicators, or the probes can be heated by external heating sources. Generally, these methods also suffer from moderate to high invasiveness, while the heating of the tumor is non-uniform, being concentrated more on the probe than elsewhere. Another way in which this can be achieved is by placing metal antennas ('seeds') in the tumor interstitium before exposure to an external magnetic field. These antennas are elongated devices made of ferromagnetic material (e.g., iron), which heat up in an alternating electromagnetic field (detailed later). In this way, the heat for hyperthermia is actually generated inside the tumor rather than filtering through from outside, and can be readily controlled by simply controlling the strength of the magnetic field. However, implanting the relatively large seeds requires an invasive procedure, which adds to the morbidity of the treatment. The seeds may focus the heating in the immediate locality of the implantation area, with resultant cold spots elsewhere in the tumor. Placing the seeds in a deep tumor may be difficult and require image guidance. Finally, it often requires large investments of money because of the requirements for special electromagnetically shielded rooms and compliance with other federal regulations. External heating can be achieved either with electromagnetic radiation (e.g., microwave, laser and radio-frequency) or high-intensity focused ultrasound, all of which transduce energy from an external source to pass through the body. One disadvantage of this method is that energy is deposited in the normal tissues along its path and can result in hot spots within these tissues. In summary, despite the beneficial effects of adding hyperthermia to the cancer management regimen, current methods to achieve it are often invasive, nonuniform or non-specific to the tumor. Clearly, there are opportunities to improve uniformity and target specificity of heat in a non-invasive or minimally invasive manner.

\section{Nanoparticles for local hyperthermia}

The foregoing discussion outlines the promises and pitfalls of established methods of generating hyperthermia. The continuing quest for a method of hyperthermia that is tumor focused, minimally invasive, and uniform has led to the investigation of nanoparticles as conduits for generating hyperthermia (Figure 2). To maximize the energy deposited in the tumor while limiting the exposure in healthy tissues, tumors can be preferentially loaded with systemically administered nanoparticles that have a high-absorption cross section for transduction of an extrinsic energy source to heat. Hyperthermia achieved by this method has several potential advantages over both global and focal hyperthermia achieved without nanoparticles. 


\section{Localization of nanoparticles}

It has been widely recognized that intravenously administered nanoparticles passively extravasate from vasculature and preferentially accumulate in tumors but not in normal tissues. This phenomenon, often referred to as the enhanced permeability and retention (EPR) effect, is a consequence of the leakiness of immature tumor neovasculature with large fenestrations and inefficient lymphatic drainage [27,28]. To ensure long circulation times of nanoparticles for efficient accumulation within tumors, evasion of non-specific reticuloendothelial capture of these particles can be achieved by rendering them 'stealth' properties via coating them with polyethylene glycol (a process known as PEGylation), dextran or other biocompatible coatings [29,30]. Additionally, nanoparticles can be decorated with targeting molecules homing to cancer-specific and cancer-associated antigens to achieve even greater tumor sensitivity [31-34]. It is to be noted that these techniques of tumor-specific localization of nanoparticles leads to tumor specific dose delivery that is independent of invasive procedures adopted in other localized dose-delivery strategies such as interstitial hyperthermia. Targeted nanoparticles also result in less nonspecific accumulation in the body, enabling a lowering of injected dose of nanoparticles for the same therapeutic effect and lessening the probability of nanoparticle-mediated toxicity.

\section{'Inside-out' hyperthermia}

All sources of heat create a temperature gradient in the tissues with the temperature falling off sharply with distance from the heat source. All external sources of heat-like focused ultrasound or infrared lamps have a narrow window of effectiveness where the skin surface is significantly affected before the temperature in the tumor reaches therapeutic levels. However, unlike all other means of external hyperthermia, the primary source of the heat the nanoparticle - is located inside the tumor. This reverses the direction of heat loss - from inside out and reduces damage to normal tissues while heating up the tumor. Furthermore, most of the nanoparticles are metallic and consequently have excellent thermal conductivity that couples and instantly transmits the heat they generate to the surrounding tumor tissue. Interstitial hyperthermia also relies on generation of heat from within the tumor but uses larger and more invasively inserted probes within tumors to achieve this.

\section{Vascular-focused hyperthermia}

Although all forms of hyperthermia seek to achieve fairly uniform temperatures globally within tumor parenchyma, a unique feature of nanoparticle-mediated hyperthermia is the location of nanoparticles in close proximity to tumor vasculature [35]. This results in a temperature gradient that is maximal at the vascular wall and falls off with distance from the perivascular space. Consequently, a global tumor parenchymal hyperthermic temperature is associated with considerably higher focal temperatures (hot spots) along tumor vasculature. By contrast, other forms of hyperthermia resulting in global tumor parenchymal hyperthermic temperatures are usually associated with focal cold spots along tumor vasculature that serve as 'heat sinks' for dissipation of heat from tumors. Consequently, the preferential vascular-focused hyperthermia achievable with nanoparticles has the advantage of not only causing heat-induced tissue damage of tumor cells but also vascular endothelia, which, in turn, sensitizes these endothelial cells and tumor cells to subsequent radiation therapy.

\section{Theranostics}

Compared to other forms of hyperthermia, nanoparticle-mediated hyperthermia has the potential to simultaneously image and treat tumors, an attribute referred to as theranostics, where therapy and diagnostics are integrated into a single platform. Since nanoparticles preferentially concentrate in tumors, detecting their presence can potentially be used as a 
means of diagnosing the presence and extent of tumors. The same nanoparticles can then be used for hyperthermia as part of tumor management. Furthermore, when the nanoparticle is decorated with tumor-specific targeting molecules that permit precise sensing and imaging of tumors, subsequent therapy (hyperthermia or thermoablation) can be image-guided.

\section{Combination with other therapies}

The fact that nanoparticles afford immense opportunities for engineering their structure more, in fact, than any other forms of injectable therapy - can be exploited to combine the hyperthermia with other therapeutic modalities. For instance, nanoparticles can be laden with or decorated with drugs, such that near-simultaneous hyperthermia and chemotherapy (drug release) can be triggered via an extrinsic energy source. Similarly, binding radioactive tracers to the nanoparticles to achieve a form of brachytherapy serves as a means of combining hyperthermia and radiation therapy.

These advantages have fueled the quest for ideal nanoparticles for achieving tumor hyperthermia. The major ones under investigation are reviewed here and include various forms of gold nanoparticles and superparamagnetic iron oxide nanoparticles (SPIONs) while carbon nanotubes (CNTs) are a more recent entrant to this field.

\section{Hyperthermia using gold nanoparticles}

\section{Characteristics of gold nanoparticles}

Many bulk metals, when reduced to a nanoscale, exhibit optical resonances of their surface plasmons, a characteristic wavelength (surface plasmon resonance) at which they strongly absorb and scatter incident light and convert resonant energy to heat. When fabricated in certain geometries, these plasmon resonances of gold can be tuned to near infrared (NIR) wavelengths, where this light penetrates deepest within human tissues due to minimal absorbance by native tissue chromophores [36]. Gold nanoparticles fabricated to have strong absorption cross sections in the NIR wavelength (up to a million-fold greater than the US FDA-approved dye indocyanine green) are also highly efficient at converting the absorbed light into heat. These photothermally activatable gold nanoparticles have many distinct advantages for clinical applications. First, gold is an inert noble metal that does not react with biological tissues and is molecularly stable. Second, long-term toxicity concerns are low due to extensive, decades-long clinical experience with the use of gold for the treatment of rheumatoid arthritis. Some caution is necessary, however, with extrapolating from larger microparticles to nanoparticles, as highlighted by a recent study that demonstrated that administration of large quantities of gold nanoparticles stimulates host immune complement activation, an effect that was dependent on other factors such as nanoparticle surface characteristics and structure [37]. Third, NIR activatable gold nanoparticles are within size regimens that permit tumor-specific accumulation via the EPR effect based on average vascular fenestrations of $60-400 \mathrm{~nm}$ in tumors. Fourth, in addition to passive accumulation in tumors via the EPR effect, surface bioconjugation for tumor-specific targeting or PEGylation for reticuloendothelial evasion can be readily performed via thiol linkages on the surface of these gold nanoparticles. Lastly, gold nanoparticles have the potential for FDA approval as a device rather than a drug, an attribute that could save significant time and money for clinical translation. Among a variety of gold nano-structures in use, the most commonly employed ones are nanoshells and nanorods.

Gold nanoshells-Nanoshells have a core of a different material coated by a thin layer of gold. The core material is dielectric, with silica being the most common material used. The resonance frequency is determined by the core:shell ratio. Gold nanoshells (GNSs) are usually close to $50-150 \mathrm{~nm}$ in the diameter and are generally moderately stable in solution, 
especially if stored at low temperatures. Silica-GNSs that are activatable by NIR light tend to be roughly $150 \mathrm{~nm}$ in diameter, with a 120 -nm diameter silica core. Extensive investigations into the safety and tolerability of GNSs have led to greater acceptance of the biocompatibility of these particles, paving the way for human clinical testing. GNSs are currently in clinical trials for head and neck cancer and prostate cancer using interstitial illumination with NIR lasers for thermoablation applications.

Gold nanorods-Gold nanorods (GNRs) are cylindrical nanoparticles made of solid gold with one dimension several times longer than the other. If the solid gold nanostructure is a sphere, the optical absorption maximum is in the region of $540 \mathrm{~nm}$ and cannot be tuned to other wavelengths. Hence this formulation is not of much use clinically. However, oblong forms, with large length:diameter aspect ratios, are tunable to higher wavelengths of light. The resonant frequency is determined by the aspect ratio, and can be calibrated to the NIR region. NIR activatable GNRs are much smaller than nanoshells, with typical sizes ranging approximately $4-5 \mathrm{~nm}$ in the longest dimension, usually with an aspect ratio of approximately 3 . To facilitate anisotropic formation of rod-shaped structures during seedmediated chemical synthesis of GNRs from spherical gold seed particles and to make them stable without aggregation in solution, a strongly charged surfactant such as cetyl trimethylammonium bromide (CTAB) is commonly employed. Since the CTAB dispersed in solution can be cytotoxic, it is often removed by serial centrifugation or dialysis, processes that involve a reduction in yield of GNRs or considerable expense. Furthermore, the CTAB on the surface of the GNRs is also shielded by a biocompatible compound such as PEG or by cross-linking a polysaccharide (chitosan) with ethylene/propylene-based block copolymers [38]. Despite some potential challenges with toxicity, GNRs - weight for weight of gold - possess a superior spectral bandwidth, are better than GNSs in heat generation from NIR light [39] and have a longer circulation half-life, improving chances for tumor accumulation.

\section{Gold nanoparticles in cancer therapy}

Hirsch et al. [40] were the first to demonstrate the effectiveness of GNS-mediated thermoablation in a mouse tumor model. While initial efforts used direct injection of GNSs into subcutaneous tumors, subsequent papers demonstrated that intravenously administered GNSs accumulated in tumors as early as $6 \mathrm{~h}$ after inoculation. Treatment of these animals with a $800 \mathrm{~mW}$ NIR laser emitting at $808 \mathrm{~nm}$ at $4 \mathrm{~W} / \mathrm{cm}^{2}$ for only $3 \mathrm{~min}$ resulted in significant survival difference over non-radiated mice [41]. Improvements in survival have been demonstrated when the gold nanoparticles are actively targeted to tumors in mice over passively targeted nanoparticles $[42,43]$. Several similar results have been demonstrated for GNRs $[44,45]$. However, despite promising results, thermoablative monotherapy of tumors with gold nanoparticles is associated with the possibility of considerable collateral damage of tissues adjacent to the tumor that are also ablated and lacks a clear advantage over established techniques such as radiofrequency ablation if interstitial laser fibers are required for photothermal ablation. Hence, combination therapy of hyperthermia (non-ablative temperatures) with other modalities is worth investigating.

In our laboratory, we have investigated the effect of modulating ionizing radiation on tumorbearing mice using GNS-mediated hyperthermia. When tumors in mice pre-treated with GNSs are subject to mild temperature hyperthermia $\left(\sim 41^{\circ} \mathrm{C}\right.$ for $\left.20 \mathrm{~min}\right)$ followed immediately by a single dose of radiation ( $10 \mathrm{~Gy}$ with $125 \mathrm{kV}$ X-rays) the tumor volume doubling time was nearly twice that with radiation alone [35]. Tumor volume doubling time is a metric used to represent tumor growth, which is independent of the initial size of the tumor; comparison of time taken for tumors to double in volume in the radiation-alone group versus tumors in a combined treatment group is a commonly employed technique for 
evaluating effectiveness of putative radiation sensitization strategies. The reasons for the in vivo response were postulated to be caused by an early increase in vascular perfusion of tumors following hyperthermia. This can be seen using dynamic contrast enhancement of MRI. However, the results also indicate that it is possible that nanoparticle-mediated hyperthermia may modulate radiation response by other mechanisms. Relatively large GNSs leak out of tumor vasculature but fail to penetrate deep into the parenchyma of tumors, remaining sequestered in the perivascular region. Heating these sequestered GNSs to generate 'inside-out' hyperthermia can possibly result in vascular disruption. This was demonstrated by a loss of microvessel density in the tumor, disruption of normal stromal architecture and regional areas of necrosis (Figures 3A \& B).

A third reason for the effectiveness of the GNS-mediated hyperthermia could be its effect on cancer stem cells. These tumor-initiating cells are hypothesized to be the primary reason for treatment failure of tumors as well as being responsible for metastatic spread. In a recent paper, it was demonstrated that radiation of breast cancer xenografts results in a reduction of tumor volume but an increase in the proportion of stem cells in the residual tumor whereas GNS-mediated hyperthermia coupled with radiation resulted in not only a greater reduction of tumor volume but also a reduction of the proportion of stem cells in the residual tumor. Furthermore, limiting dilution transplantation of the cancer cells from residual tumors following combined treatment resulted in a lower frequency of tumor formation than similar transplantation of cells from residual tumors following radiation alone (Figure 3c).

Overcoming the inherent radio-resistance of cancer stem cells, which are thought to be the primary reason for therapy failure, provide another important rationale for the addition of hyperthermia to radiation therapy of tumors [46].

\section{Hyperthermia using SPIONs Characteristics of SPIONs}

Magnetic materials are either permanent magnets (ferromagnetic, like iron) or which only demonstrate magnetism under the influence of an external magnetic field (paramagnetic). A new property is obtained when ferromagnetic materials are fabricated on the nanoscale that of superparamagnetism. On the nanoscale, the ferromagnetic nanoparticles can randomly flip the orientation of their magnetic dipoles, a phenomenon that makes them appear paramagnetic. However, under the influence of an external magnetic field, they align in the direction of the field, and the resultant magnetic susceptibility is orders of magnitude higher than that of standard paramagnetic material. When the material for the nanoparticles is iron oxide (the most common), the nanoparticles are called SPIONs.

In theory, alternating the external magnetic field rapidly would cause the particles to rapidly flip their magnetic polarity. However, there is some hysteretic loss involved in the flipping, which manifests as heat. If a tissue (say, a tumor) is pre-loaded with SPIONs and then subjected to alternating magnetic field (AMF), it will heat up. Several factors will affect the extent of this heating - the magnitude of the field, the size and characteristic of the SPIONs, the depth of the tumor within the body and the concentration of SPIONs in the tumor.

The first instance of the treatment of cancer using magnetic iron oxide particles was probably in 1957 [47]. Since then the synthesis and design of SPIONs have progressed much. At the heart of the SPION is the magnetic core of iron oxide. Synthesis is usually by the precipitation of iron salts in the presence of various chemicals, for example ammonia [48], sodium nitrate/sodium hydroxide [49] or by photochemical methods [50].

Bare SPIONs are rapidly cleared from the blood after intravenous injection and accumulate in the liver, spleen, and lymph nodes. When biocompatible dextran magnetite nanoparticles 
are injected intravenously in rats, clearance was initially very rapid (half-life of $10 \mathrm{~min}$ ) followed by a slower decrease (half-life of $92 \mathrm{~min}$ ) [51]. In the first $2 \mathrm{~h}$, the particles spread throughout the body including the liver and the spleen [52]. However, over time, the particles are scavenged by the reticuloendothelial system and accumulate in the macrophages of the splenic marginal zone, where there is a steady and rapid increase in accumulated particles for up to $48 \mathrm{~h}$, followed by a decrease until the end of the observed period of 25 days. The iron oxide cores sequester as granules in the Kupffer cells of the liver, leading to a slow increase in accumulated amount.

To enable tumor accumulation, SPIONs need to be coated with or embedded in a polymer shell. The most common method for this is to embed the SPIONs in a polymeric nanoparticle that is hydrophilic. A common polymer for this purpose is dextran [53], although other polymeric blends, such as $\beta$-cyclodextrin and pluronic polymer combination [48], and chitosan [54], can also be used. Apart from polymers, iron/iron oxide core-shell nanoparticles are also popular $[55,56]$. Silica is a popular coat for many varieties of nanoparticles, including SPIONs $[49,57]$. However, the resultant particles are often microspheres rather than nanoparticles, with diameters of approximately $20-30 \mu \mathrm{m}$. These are used as in situ implants into tumors rather than as intravenous formulations.

Subsequently, to enhance circulation half-life, the dextran coat is often covered by a monolayer of PEG [55]. Finally, targeting molecules can be attached to enhance specificity of uptake by cancer cells. For example, folic acid $[54,58]$ can be attached to target folate receptors in several solid tumors. Addition of folic acid was found to increase the cytotoxicity against the folate receptor over-expressing KB cells but had little adverse impact on the A549 cells, which do not possess these receptors. In addition, a novel way of coating the SPIONs is the use of gold [59]. The thin gold shell can itself be heated up by AMF, thus adding to the hyperthermic effect. Indeed, gold-coated SPIONs demonstrated several-fold higher increases in heat release compared with SPIONs alone. Furthermore, SPION-mediated hyperthermia can be combined with other strategies to further enhance the therapeutic outcome. For example, the SPIONs can be coated with thermosensitive polymers that are loaded with anti-cancer drugs $[48,58]$. Application of AMF after accumulation of the SPIONs in tumors would raise the local temperature of the SPIONs sufficiently to exceed the critical temperature of the polymers, resulting in drug release.

\section{SPIONs in cancer therapy}

Delivery of adequate amounts of SPIONs into tumors is a prerequisite for successful hyperthermia. These can be delivered to possible nests of metastatic cells in lymph nodes by direct injection into the lymphatic channels draining into the lymph nodes [47].

Subsequently, a number of investigators injected the SPIONs directly into the tumor as proof-of-principle experiments. The SPIONs can be injected in solution, or loaded into hydrogels and organogels [57].

However, this more invasive method of SPION delivery is not favorable, owing to obvious difficulties in translating to clinical usage. The favored delivery route is intravenous; however, successful concentration of nanoparticles into tumors by this route is not easily achievable. A novel route of delivery is by loading the SPIONs into cells [56]. In the experiment described, tumor-tropic neural progenitor cells were loaded with SPIONs and transplanted into melanoma-bearing mice, whence the cells made their way into the tumors. Subsequent AMF exposure resulted in tumor regression.

To achieve adequate concentrations in the tumor, SPIONs are sometimes conjugated to targeting molecules. Triton BioSystems, Inc. (Chelmsford, MA) have developed one such system of AMF-responsive nanoparticles and targeted these to cancerous tissues by 
conjugating with monoclonal antibodies. The combination of molecular targeting and the focused AMF field aims to achieve highly sensitive tumor hyperthermia.

The use of heat for cancer can be ablative or sub-ablative. When sub-ablative, it can be used either as a standalone therapy to delay tumor growth [50] or as a means of radiosensitization [46]. The degree of heating of the tumor can be controlled by the AMF field. However, increasing the current in the AMF generator, and thus increasing the AMF field strength, generates heat in tissues by inductive tissue heating from eddy current losses. This is independent of the presence of SPIONs and affects normal tissues in the AMF field. The raised temperature in the normal tissues limits the extent to which the AMF field can be increased. In mouse experiments, for example, radiofrequency exposures at $25 \mathrm{~kW}$ for 20 min raised the tumor core temperature to $47^{\circ} \mathrm{C}[50]$.

Another limitation of the use of SPIONs is the toxicity to normal cells in the absence of AMF. Biocompatibility studies with dextran and citric acid-coated SPIONs on human umbilical-vein endothelial cells [53] demonstrated the expected endocytotic uptake by the cells, followed by cell death through suspected apoptotic pathways. Even at concentrations as low as $0.1 \mathrm{mM}$, the coated SPIONs managed to inhibit migration/invasion functions of the cells. While cytotoxic effects are strongly dependent on the coating/matrix of the SPIONs, the results demonstrated the necessity of thorough cytotoxicity evaluation of any proposed therapy involving these nanoparticles.

\section{Hyperthermia with CNTs}

\section{Characteristics of CNTs}

CNTs are unique, (almost) 1D nanomaterials composed of single sheets of graphene rolled into the shape of a tube. Since the aspect ratio and the direction of rolling can be controlled at synthesis, the optical properties can also be tuned. Similar to gold nanoparticles, singlewalled CNTs (SWCNTs) also strongly absorb electromagnetic waves to generate heat. Instead of plasmon resonance, absorption of light by CNTs results in excitation of electronic transitions within the nanostructure with relaxation resulting in amplified vibrational modes within the carbon lattice. There is neither an absorption threshold nor is the absorption a continuous function of energy, but absorption occurs as discrete tunable and sharp spikes. Also, unlike gold, CNTs can absorb over a broad frequency range including visible light, NIR light and even radiofrequency irradiation. The extinction coefficient of such absorption is much higher than that of common tissue chromophores, such as melanins, hemoglobin and water [49].

CNTs are very thin cylinders of graphene, which can be synthesized to considerable variations in aspect ratios. The diameter of the tubes can vary from the nano- to the microscales [60]. Usually the cylinders are SWCNTs, but more recently multi-walled CNTs (MWCNTs) have also been investigated for their optical properties. They have been shown to have enhanced absorption cross-sections when compared with SWCNTs [61].

\section{CNTs in cancer therapy}

Following in the footsteps of the GNRs and SPIONs, the enhanced optical cross section of CNTs has been investigated for photothermal ablation of cancer cells [61]. This has been demonstrated in vitro using SWCNTs [61-63]. Tumor destruction in mouse models of cancer have also been demonstrated using intratumoral injection of SWCNTs followed by NIR irradiation for only $3 \mathrm{~min}$ [64]. Similar to gold, not only NIR, but also radiofrequency fields have been used to generate hyperthermia using SWCNTs. The required radiofrequency field was in the megahertz region $(13.56 \mathrm{mHz})$. The in vitro study was succeeded by in vivo studies using direct intratumoral injection of the nanotubes. 
Hyperthermia using the radiofrequency field resulted in complete necrosis of tumors [65]. Not only SWCNTs, MWCNTs have also been demonstrated to be effective in tumor ablation using NIR light. However, unlike SWCNTs, MWCNTs achieve thermal ablation of tumors after exposure to only a $30 \mathrm{~s}$ burst of NIR light at a relatively low laser power of 3 $\mathrm{W} / \mathrm{cm}^{2}[66]$.

The major stumbling block to more widespread adoption of CNTs is a concern regarding their toxicity. Exposure of mesothelial and pleural lining to CNTs can result in granulomas reminiscent of mesotheliomas arising from asbestos exposure [67]. Several factors, including their propensity to aggregate, and the nature of the surface and functional groups attached thereof, affect the toxicity of these nanostructures. Although it has been demonstrated that at low doses CNTs are non-toxic to mice, more detailed studies are necessary prior to adopting these for clinical use.

There are reports of other nanoparticles beyond these three types, which can potentially be exploited for hyperthermia. For example, fluorescent quantum dots [68], silver and zinc nanoparticles and lanthanum manganite particles with impregnated silver ions [69] have been explored. However, the general principles discussed for the three nanoparticles hold true in most cases.

\section{Conclusion \& future perspective}

Hyperthermia results in increasing perfusion of tumors. This results in less hypoxic areas and a better response to radiation, while also aiding chemotherapy. However, beyond these traditional effects, nanoparticle-mediated hyperthermia is proving to have additional roles to play in cancer therapy - from disruption of microvasculature to sensitization of recalcitrant cancer stem cells to radiation. All this makes quick addition of this therapeutic modality to the oncologist's repetoire an important priority.

However, despite the potential role that hyperthermia can play in cancer management it has not been adequately exploited clinically. There are several reasons for this. Historical methods of achieving global hyperthermia were cumbersome, non-standardized and nonspecific. More recent methods of generating hyperthermia are still frequently invasive and/ or result in non-uniform temperature elevations within tumors and possible hot spots in surrounding normal tissues. The breakthrough has been the introduction of injectable nanoparticles like SPIONs, GNSs and CNTs that made it possible to achieve ablative temperatures inside highly localized areas of the body, while maintaining other areas at normal or near-normal temperatures. Nanoparticles provide a promising alternative to the older techniques to attain tumor hyperthermia.

However, there are several challenges facing the use of nanoparticles for tumor hyperthermia. A major problem is adequacy and uniformity of accumulation of nanoparticles at the tumor site. Even with very small nanoparticles, uniform temperature throughout the core and mantle of the tumor is still difficult to obtain [16]. Nanoparticles do not readily penetrate uniformly into the poorly vascularized tumor core. Other avenues need to be explored to find means of raising temperature uniformly in the core.

A second issue hampering the clinical translation of nanoparticles is the issue of quality control. Nanoparticles made in the laboratory often suffer from intra-batch and inter-batch variations in size and composition. As nanoparticles become more complex in composition, the risk of variation increases proportionally. Size distribution of nanoparticles are typically assessed using dynamic light scattering (e.g., with the zetasizer), but this provides an estimate of hydrodynamic radius rather than actual diameter. The actual diameter can be seen from electron microscopy, but only a very small number of nanoparticles can be seen 
per image and extrapolating size of the full sample from the images can lead to errors. When nanoparticles are coated with targeting ligands, presence and quantification of the ligands, and in some cases testing of the functional capabilities, need to be done. Obviously, scaling up of production for industry will be an issue for complex nanoparticles. However, despite these obstacles, better methods of nanoparticle synthesis are constantly being reported. Most commercially available nanoparticles (e.g., the GNSs from Nanospectra, Inc.) are now available in batches with less than $1 \%$ variation in diameters. It is expected that in the near future we will have more complex nanoparticles being produced with equivalent quality controls.

A third obstacle in the clinical acceptance of nanoparticles is biocompatibility. The problem might be considered to be twofold: one of immediate toxicity and one of delayed effects of retained nanoparticles. Immediate and direct toxicity issues can be resolved using standardized testing on appropriate animal models. Indeed, most nanoparticles have been shown to be relatively nontoxic in the doses required for therapeutic effect. Gold, in particular, has a long history of usage in medical conditions, and GNS have been demonstrated to be safe in the administered quantities. As a result, clinical trials have been launched for human testing. However, GNRs have a unique challenge; as mentioned above, the persistent $\mathrm{CTAB}$ in the solution needs to be removed or neutralized before clinical usage is possible. The more difficult challenge is the question of the long-term fate of the nanoparticles sequestered in the body. Hepatic and splenic macrophages ingest and store much of the injected nanoparticles; the long-term consequences of this storage are as yet uncertain.

Similar to other treatment modalities, such as chemotherapy and radiation, hyperthermia is most effective when confined to the tumor. While enhanced permeation and retention allows passive accumulation at tumors, there is also concurrent accumulation in some other tissues, most notably the liver. This makes use of this technique less effective when dealing with tumors of the liver and surrounding areas. Accumulation at other areas, though less prominent, argues for thorough investigation of the biodistribution for each particle proposed for clinical use.

In conclusion, nanoparticles hold promise as a novel means of generating hyperthermia with distinct advantages over traditional methods. Comprehensive toxicity evaluations, optimized methods to ensure uniform, adequate and specific intratumoral delivery and greater dose deposition at tumors are the challenges that face effective clinical exploitation of nanoparticle-mediated hyperthermia in tumor treatment.

\section{Key Terms}

\section{Antenna \\ Radiofrequency and microwaves}

Theranostic

Surface plasmon resonance
An antenna (as used in medical hyperthermia) is a small metal piece which absorbs radio waves and consequently heats up

Radiofrequency waves are electromagnetic waves with the rate of oscillation in the range of approximately $3 \mathrm{kHz}$ to 300 $\mathrm{GHz}$; microwaves are a subset at the higher energy end of this spectrum $(300 \mathrm{MHz}$ to $300 \mathrm{GHz}$ )

A combination of the terms therapeutic and diagnostic; usually refers to a device which can perform both functions

Plasmons are quantized oscillations of free electrons. These electron waves propagate along the surface of metal 


\section{Core:shell ratio}

Superparamagnetism

Graphene nanoparticles, and can resonate when photons of certain wavelength are incident on them

Ratio of the diameter of the core to the thickness of the shell of core shell nanoparticles. A higher core:shell ratio means a thinner shell

Small nanometer-sized ferro- and ferri-magnetic particles demonstrate no magnetization in the absence of a magnetic field (unlike similar material in the meso- and macroscopic scales). These are magnetized in the presence of a magnetic field (such as paramagnetic material, e.g., gadolinium) but much more strongly than compared with conventioanl paramagnets

Graphene is an allotrope of carbon, where the carbon atoms are bonded in a honeycomb pattern to form 2D crystalline planar sheets. The sheet can be rolled into tubes (carbon nanotubes) or stacked into a 3D sheet (graphite)

\section{Bibliography}

1. Coley WB II. Contribution to the Knowledge of Sarcoma. Ann Surg. 1891; 14(3):199-220.

2. Friedenthal E, Mendecki J, Botstein C, Sterzer F, Nowogrodzki M, Paglione R. Some practical considerations for the use of localized hyperthermia in the treatment of cancer. J Microw Power. 1981; 16(2):199-204. [PubMed: 6915106]

3. Lele PP. Induction of deep, local hyperthermia by ultrasound and electromagnetic fields: problems and choices. Radiat Environ Biophys. 1980; 17(3):205-217. [PubMed: 7443976]

4. Magin RL, Johnson RK. Effects of local tumor hyperthermia on the growth of solid mouse tumors. Cancer Res. 1979; 39(11):4534-4539. [PubMed: 498085]

5. Kim JH, Hahn EW, Ahmed SA. Combination hyperthermia and radiation therapy for malignant melanoma. Cancer. 1982; 50(3):478-482. [PubMed: 7093890]

6. Luk KH, Francis ME, Perez CA, Johnson RJ. Combined radiation and hyperthermia: comparison of two treatment schedules based on data from a registry established by the Radiation Therapy Oncology Group (RTOG). Int J Radiat Oncol Biol Phys. 1984; 10(6):801-809. [PubMed: 6735765]

7. Stewart JR, Gibbs FA Jr. Hyperthermia in the treatment of cancer. Perspectives on its promise and its problems. Cancer. 1984; 54(11 Suppl):2823-2830. [PubMed: 6388817]

8. Thrall DE. Clinical requirements for localized hyperthermia in the patient. Radiat Environ Biophys. 1980; 17(3):229-232. [PubMed: 7443978]

9. Doss JD, Mccabe CW. A technique for localized heating in tissue: an adjunct to tumor therapy. Med Instrum. 1976; 10(1):16-21. [PubMed: 1250180]

10. Irish CE, Brown J, Galen WP, et al. Thermoradiotherapy for persistent cancer in previously irradiated fields. Cancer. 1986; 57(12):2275-2279. [PubMed: 3697929]

11. Seegenschmiedt MH, Sauer R, Miyamoto C, Chalal JA, Brady LW. Clinical experience with interstitial thermoradiotherapy for localized implantable pelvic tumors. Am J Clin Oncol. 1993; 16(3):210-222. [PubMed: 8338055]

12. Bass H, Moore JL, Coakley WT. Lethality in mammalian cells due to hyperthermia under oxic and hypoxic conditions. Int J Radiat Biol Relat Stud Phys Chem Med. 1978; 33(1):57-67. [PubMed: 304850]

13. Storm FK. Clinical hyperthermia and chemotherapy. Radiol Clin North Am. 1989; 27(3):621-627. [PubMed: 2648463]

14. Urano M, Kuroda M, Nishimura Y. For the clinical application of thermochemotherapy given at mild temperatures. Int J Hyperthermia. 1999; 15(2):79-107. [PubMed: 10323618] 
15. Shakil A, Osborn JL, Song CW. Changes in oxygenation status and blood flow in a rat tumor model by mild temperature hyperthermia. Int J Radiat Oncol Biol Phys. 1999; 43(4):859-865. [PubMed: 10098442]

16. Johannsen M, Thiesen B, Wust P, Jordan A. Magnetic nanoparticle hyperthermia for prostate cancer. Int J Hyperthermia. 2010; 26(8):790-795. [PubMed: 20653418]

17. Hurwitz MD, Hansen JL, Prokopios-Davos S, et al. Hyperthermia combined with radiation for the treatment of locally advanced prostate cancer: long-term results from Dana-Farber Cancer Institute study 94-153. Cancer. 2011; 117(3):510-516. [PubMed: 20886629]

18. Moros EG, Penagaricano J, Novak P, Straube WL, Myerson RJ. Present and future technology for simultaneous superficial thermoradiotherapy of breast cancer. Int J Hyperthermia. 2010; 26(7): 699-709. [PubMed: 20849263]

19. Van Den Berg CA, Van De Kamer JB, De Leeuw AA, et al. Towards patient specific thermal modelling of the prostate. Phys Med Biol. 2006; 51(4):809-825. [PubMed: 16467580]

20. Zagar TM, Oleson JR, Vujaskovic Z, et al. Hyperthermia combined with radiation therapy for superficial breast cancer and chest wall recurrence: a review of the randomised data. Int $\mathrm{J}$ Hyperthermia. 2010; 26(7):612-617. [PubMed: 20849256]

21. Franckena M, Van Der Zee J. Use of combined radiation and hyperthermia for gynecological cancer. Curr Opin Obstet Gynecol. 2010; 22(1):9-14. [PubMed: 20019611]

22. Vasanthan A, Mitsumori M, Park JH, et al. Regional hyperthermia combined with radiotherapy for uterine cervical cancers: a multi-institutional prospective randomized trial of the international atomic energy agency. Int J Radiat Oncol Biol Phys. 2005; 61(1):145-153. [PubMed: 15629605]

23. Huilgol NG, Gupta S, Sridhar CR. Hyperthermia with radiation in the treatment of locally advanced head and neck cancer: a report of randomized trial. J Cancer Res Ther. 2010; 6(4):492496. [PubMed: 21358087]

24. Huilgol NG, Gupta S, Dixit R. Chemoradiation with hyperthermia in the treatment of head and neck cancer. Int J Hyperthermia. 2010; 26(1):21-25. [PubMed: 20100049]

25. Chang E, Alexander HR, Libutti SK, et al. Laparoscopic continuous hyperthermic peritoneal perfusion. J Am Coll Surg. 2001; 193(2):225-229. [PubMed: 11491455]

26. Feldman AL, Libutti SK, Pingpank JF, et al. Analysis of factors associated with outcome in patients with malignant peritoneal mesothelioma undergoing surgical debulking and intraperitoneal chemotherapy. J Clin Oncol. 2003; 21(24):4560-4567. [PubMed: 14673042]

27. Maeda $H$. The enhanced permeability and retention (EPR) effect in tumor vasculature: the key role of tumor-selective macromolecular drug targeting. Adv Enzyme Regul. 2001; 41:189-207. [PubMed: 11384745]

28. Maeda H, Wu J, Sawa T, Matsumura Y, Hori K. Tumor vascular permeability and the EPR effect in macromolecular therapeutics: a review. J Control Release. 2000; 65(1-2):271-284. [PubMed: 10699287]

29. Kah JC, Wong KY, Neoh KG, et al. Critical parameters in the pegylation of gold nanoshells for biomedical applications: an in vitro macrophage study. J Drug Target. 2009; 17(3):181-193. [PubMed: 19016072]

30. Wang J, Sui M, Fan W. Nanoparticles for tumor targeted therapies and their pharmacokinetics. Curr Drug Metab. 2010; 11(2):129-141. [PubMed: 20359289]

31. El-Sayed IH, Huang X, El-Sayed MA. Selective laser photo-thermal therapy of epithelial carcinoma using anti-EGFR antibody conjugated gold nanoparticles. Cancer Lett. 2006; 239(1): 129-135. [PubMed: 16198049]

32. Waldman SA, Fortina P, Surrey S, Hyslop T, Kricka LJ, Graves DJ. Opportunities for nearinfrared thermal ablation of colorectal metastases by guanylyl cyclase C-targeted gold nanoshells. Future Oncol. 2006; 2(6):705-716. [PubMed: 17155897]

33. Patra CR, Bhattacharya R, Mukhopadhyay D, Mukherjee P. Fabrication of gold nanoparticles for targeted therapy in pancreatic cancer. Adv Drug Deliv Rev. 2010; 62(3):346-361. [PubMed: 19914317]

34. Hosta-Rigau L, Olmedo I, Arbiol J, Cruz LJ, Kogan MJ, Albericio F. Multifunctionalized gold nanoparticles with peptides targeted to gastrin-releasing peptide receptor of a tumor cell line. Bioconjug Chem. 2010; 21(6):1070-1078. [PubMed: 20476781] 
35. Diagaradjane P, Shetty A, Wang JC, et al. Modulation of in vivo tumor radiation response via gold nanoshell-mediated vascular-focused hyperthermia: characterizing an integrated antihypoxic and localized vascular disrupting targeting strategy. Nano Lett. 2008; 8(5):1492-1500. [PubMed: 18412402]

36. Hirsch LR, Gobin AM, Lowery AR, et al. Metal nanoshells. Ann Biomed Eng. 2006; 34(1):15-22. [PubMed: 16528617]

37. Hulander M, Hong J, Andersson M, et al. Blood interactions with noble metals: coagulation and immune complement activation. ACS Appl Mater Interfaces. 2009; 1(5):1053-1062. [PubMed: 20355891]

38. Choi WI, Kim JY, Kang C, Byeon CC, Kim YH, Tae G. Tumor regression in vivo by photothermal therapy based on gold-nanorod-loaded, functional nanocarriers. ACS Nano. 2011; 5(3):19952003. [PubMed: 21344891]

39. Von Maltzahn G, Park JH, Agrawal A, et al. Computationally guided photothermal tumor therapy using long-circulating gold nanorod antennas. Cancer Res. 2009; 69(9):3892-3900. [PubMed: 19366797]

40. Hirsch LR, Stafford RJ, Bankson JA, et al. Nanoshell-mediated near-infrared thermal therapy of tumors under magnetic resonance guidance. Proc Natl Acad Sci USA. 2003; 100(23):13549_ 13554. [PubMed: 14597719]

41. O'neal DP, Hirsch LR, Halas NJ, Payne JD, West JL. Photo-thermal tumor ablation in mice using near infrared-absorbing nanoparticles. Cancer Lett. 2004; 209(2):171-176. [PubMed: 15159019]

42. Bernardi RJ, Lowery AR, Thompson PA, Blaney SM, West JL. Immunonanoshells for targeted photothermal ablation in medulloblastoma and glioma: an in vitro evaluation using human cell lines. J Neurooncol. 2008; 86(2):165-172. [PubMed: 17805488]

43. Cheng FY, Chen CT, Yeh CS. Comparative efficiencies of photothermal destruction of malignant cells using antibody-coated silica-Au nanoshells, hollow Au/Ag nanospheres and Au nanorods. Nanotechnology. 2009; 20(42):425104. [PubMed: 19779243]

44. Dickerson EB, Dreaden EC, Huang X, et al. Gold nanorod assisted near-infrared plasmonic photothermal therapy (PPTT) of squamous cell carcinoma in mice. Cancer Lett. 2008; 269(1):5766. [PubMed: 18541363]

45. Skrabalak SE, Au L, Lu X, Li X, Xia Y. Gold nanocages for cancer detection and treatment. Nanomedicine (Lond). 2007; 2(5):657-668. [PubMed: 17976028]

46. Atkinson RL, Zhang M, Diagaradjane P, et al. Thermal enhancement with optically activated gold nanoshells sensitizes breast cancer stem cells to radiation therapy. Sci Transl Med. 2010; 2(55): $55 \mathrm{ra79}$.

47. Gilchrist RK, Medal R, Shorey WD, Hanselman RC, Parrott JC, Taylor CB. Selective inductive heating of lymph nodes. Ann Surg. 1957; 146(4):596-606. [PubMed: 13470751]

48. Yallapu MM, Othman SF, Curtis ET, Gupta BK, Jaggi M, Chauhan SC. Multi-functional magnetic nanoparticles for magnetic resonance imaging and cancer therapy. Biomaterials. 2011; 32(7): 1890-1905. [PubMed: 21167595]

49. Huang N, Wang H, Zhao J, Lui H, Korbelik M, Zeng H. Single-wall carbon nanotubes assisted photothermal cancer therapy: animal study with a murine model of squamous cell carcinoma. Lasers Surg Med. 42(9):638-648. [PubMed: 20949599]

50. Elsherbini AA, Saber M, Aggag M, El-Shahawy A, Shokier HA. Magnetic nanoparticle-induced hyperthermia treatment under magnetic resonance imaging. Magn Reson Imaging. 2011; 29(2): 272-280. [PubMed: 21145190]

51. Majumdar S, Zoghbi SS, Gore JC. Pharmacokinetics of superparamagnetic iron-oxide MR contrast agents in the rat. Invest Radiol. 1990; 25(7):771-777. [PubMed: 2391194]

52. Okon E, Pouliquen D, Okon P, et al. Biodegradation of magnetite dextran nanoparticles in the rat. A histologic and biophysical study. Lab Invest. 1994; 71(6):895-903. [PubMed: 7807971]

53. Wu X, Tan Y, Mao H, Zhang M. Toxic effects of iron oxide nanoparticles on human umbilical vein endothelial cells. Int J Nanomedicine. 2010; 5:385-399. [PubMed: 20957160]

54. Fan C, Gao W, Chen Z, et al. Tumor selectivity of stealth multi-functionalized superparamagnetic iron oxide nanoparticles. Int J Pharm. 2011; 404(1-2):180-190. [PubMed: 21087660] 
55. Balivada S, Rachakatla RS, Wang H, et al. A/C magnetic hyperthermia of melanoma mediated by iron(0)/iron oxide core/shell magnetic nanoparticles: a mouse study. BMC Cancer. 2010; 10:119. [PubMed: 20350328]

56. Rachakatla RS, Balivada S, Seo GM, et al. Attenuation of mouse melanoma by A/C magnetic field after delivery of bi-magnetic nanoparticles by neural progenitor cells. ACS Nano. 2010; 4(12): 7093-7104. [PubMed: 21058696]

57. Le Renard PE, Jordan O, Faes A, et al. The in vivo performance of magnetic particle-loaded injectable, in situ gelling, carriers for the delivery of local hyperthermia. Biomaterials. 2010; 31(4):691-705. [PubMed: 19878991]

58. Hayashi K, Ono K, Suzuki H, et al. High-frequency, magnetic-field-responsive drug release from magnetic nanoparticle/organic hybrid based on hyperthermic effect. ACS Appl Mater Interfaces. 2010; 2(7):1903-1911. [PubMed: 20568697]

59. Mohammad F, Balaji G, Weber A, Uppu RM, Kumar CS. Influence of gold nanoshell on hyperthermia of superparamagnetic iron oxide nanoparticles (SPIONs). J Phys Chem C Nanomater Interfaces. 2010; 1(20):3141-3146. [PubMed: 21103390]

60. Zheng LX, O'connell MJ, Doorn SK, et al. Ultralong single-wall carbon nanotubes. Nat Mater. 2004; 3(10):673-676. [PubMed: 15359345]

61. Kam NW, O’Connell M, Wisdom JA, Dai H. Carbon nanotubes as multifunctional biological transporters and near-infrared agents for selective cancer cell destruction. Proc Natl Acad Sci USA. 2005; 102(33):11600-11605. [PubMed: 16087878]

62. Biris AS, Boldor D, Palmer J, et al. Nanophotothermolysis of multiple scattered cancer cells with carbon nanotubes guided by time-resolved infrared thermal imaging. J Biomed Opt. 2009; 14(2): 021007. [PubMed: 19405720]

63. Torti SV, Byrne F, Whelan O, et al. Thermal ablation therapeutics based on CN(x) multi-walled nanotubes. Int J Nanomedicine. 2007; 2(4):707-714. [PubMed: 18203437]

64. Moon HK, Lee SH, Choi HC. In vivo near-infrared mediated tumor destruction by photothermal effect of carbon nanotubes. ACS Nano. 2009; 3(11):3707-3713. [PubMed: 19877694]

65. Gannon CJ, Cherukuri P, Yakobson BI, et al. Carbon nanotube-enhanced thermal destruction of cancer cells in a noninvasive radiofrequency field. Cancer. 2007; 110(12):2654-2665. [PubMed: 17960610]

66. Burke A, Ding X, Singh R, et al. Long-term survival following a single treatment of kidney tumors with multiwalled carbon nanotubes and near-infrared radiation. Proc Natl Acad Sci USA. 2009; 106(31):12897-12902. [PubMed: 19620717]

67. Poland CA, Duffin R, Kinloch I, et al. Carbon nanotubes introduced into the abdominal cavity of mice show asbestos-like pathogenicity in a pilot study. Nat Nanotechnol. 2008; 3(7):423-428. [PubMed: 18654567]

68. Glazer ES, Curley SA. Radiofrequency field-induced thermal cytotoxicity in cancer cells treated with fluorescent nanoparticles. Cancer. 2010; 116(13):3285-3293. [PubMed: 20564640]

69. Melnikov OV, Gorbenko OY, Markelova MN, et al. Ag-doped manganite nanoparticles: new materials for temperature-controlled medical hyperthermia. J Biomed Mater Res A. 2009; 91(4): 1048-1055. [PubMed: 19127514]

70. Fuller KJ, Issels RD, Slosman DO, Guillet JG, Soussi T, Polla BS. Cancer and the heat-shock response. Eur J Cancer. 1994; 30A(12):1884-1891. [PubMed: 7880622]

71. Harmon BV, Takano YS, Winterford CM, Gobe GC. The role of apoptosis in the response of cells and tumours to mild hyperthermia. Int J Radiat Biol. 1991; 59(2):489-501. [PubMed: 1671698] 


\section{Executive summary}

- Hyperthermia, a small rise in tumor temperature, alters the cancer cells (especially the cancer stem cells) and the microenvironment to increase susceptibility to radiation and chemotherapy.

- Specific and uniform elevation of tumor temperature is difficult with wholebody and regional hyperthermia. Luminal and interstitial local hyperthermia are invasive, while external radiation may cause skin burns.

- Preferential loading of tumors with systemically administered, energytransducing nanoparticles allows tumor specificity, heating from within the tumor, vascular disruption, simultaneous imaging and therapy (theranostics) and single-platform combination with other therapies.

- Three major nanoparticles for generating hyperthermia are gold nanoparticles, superparamagnetic iron oxide nanoparticles (SPIONs) and carbon nanotubes (CNTs).

- Gold nanoparticles with strong absorption cross sections in the near infrared wavelength due to surface plasmon resonance, efficiently generate heat upon illumination and are inert, stable and readily conjugated to biomolecules for specific tumor targeting. Two major types are nanoshells and nanorods.

- SPIONs heat up rapidly in an alternating magnetic field depending on the field strength and frequency, particle concentration and characteristics and the depth of the tumor within the body.

- CNTs are largely unidimensional nanomaterials composed of rolled sheets of graphene with tunable absorption of electromagnetic waves over a broad frequency range to generate heat.

- Obstacles to clinical exploitation of hyperthermia can potentially be overcome with nanoparticles providing more efficient methods of generating uniform heating in tumors with minimal effect on normal tissue.

- Challenges facing nanoparticle-mediated tumor hyperthermia include inadequacy and non-uniformity of accumulation in tumors, especially the core; lack of quality control in production; perceived toxicity issues; concurrent accumulation in the liver and other tissues; and thermal modeling/dosimetry. 
External hyperthermia

(e.g., focused ultrasound infrared and AMF)

Figure 1. Methods of achieving tumor hyperthermia

AMF: Alternating magnetic field

Nanoparticle-based hyperthermia (intravenously administered targeted nanoparticles accumulate in tumors

and act as foci for heat

by transducing energy delivered externally)
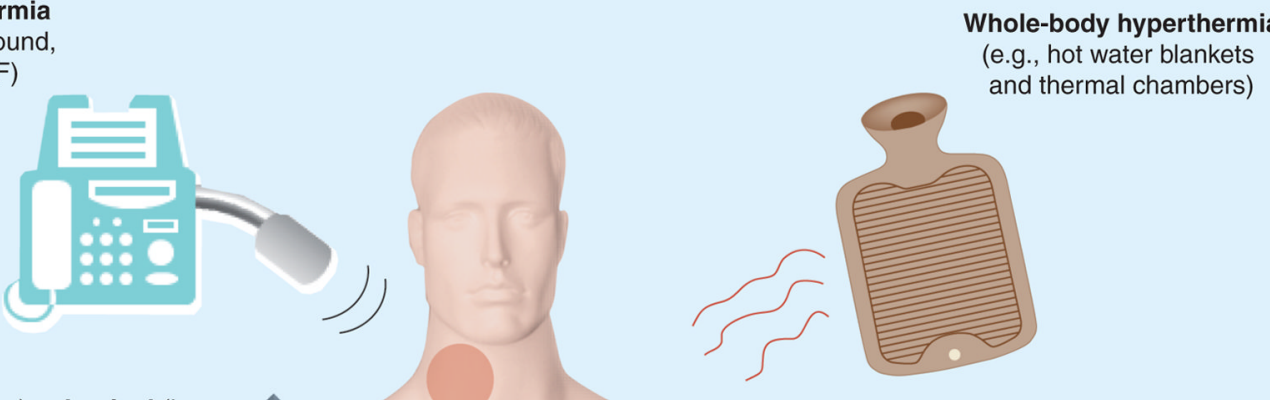

Interstitial (in the tumor) or luminal (in a based on location of inserted probes/applicators
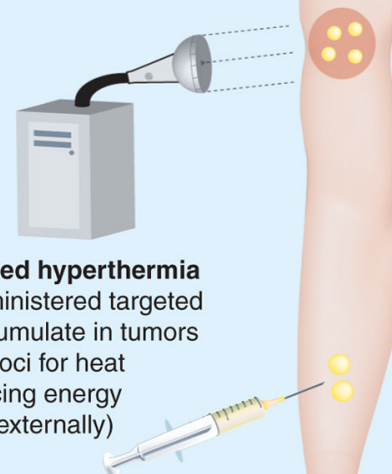

Therapeutic Delivery (C) Future Science Group (20II)

\section{Regional hyperthermia} (perfusion with hot liquids and warming blood ex vivo) 


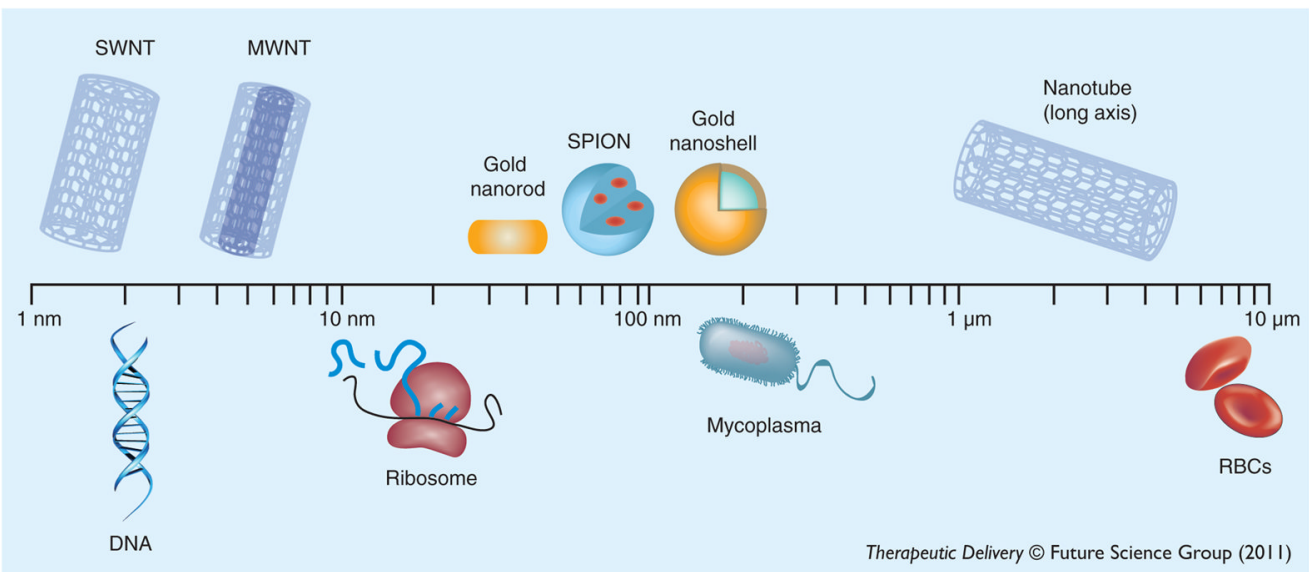

Figure 2. Relative scale of synthetic nanomaterials as compared with naturally occurring biomacromolecules and cells

MWNT: Multiwalled carbon nanotubes; RBC: Red blood cell; SPION: Superparamagnetic iron oxide nanoparticle; SWNT: Single-walled carbon nanotubes. 


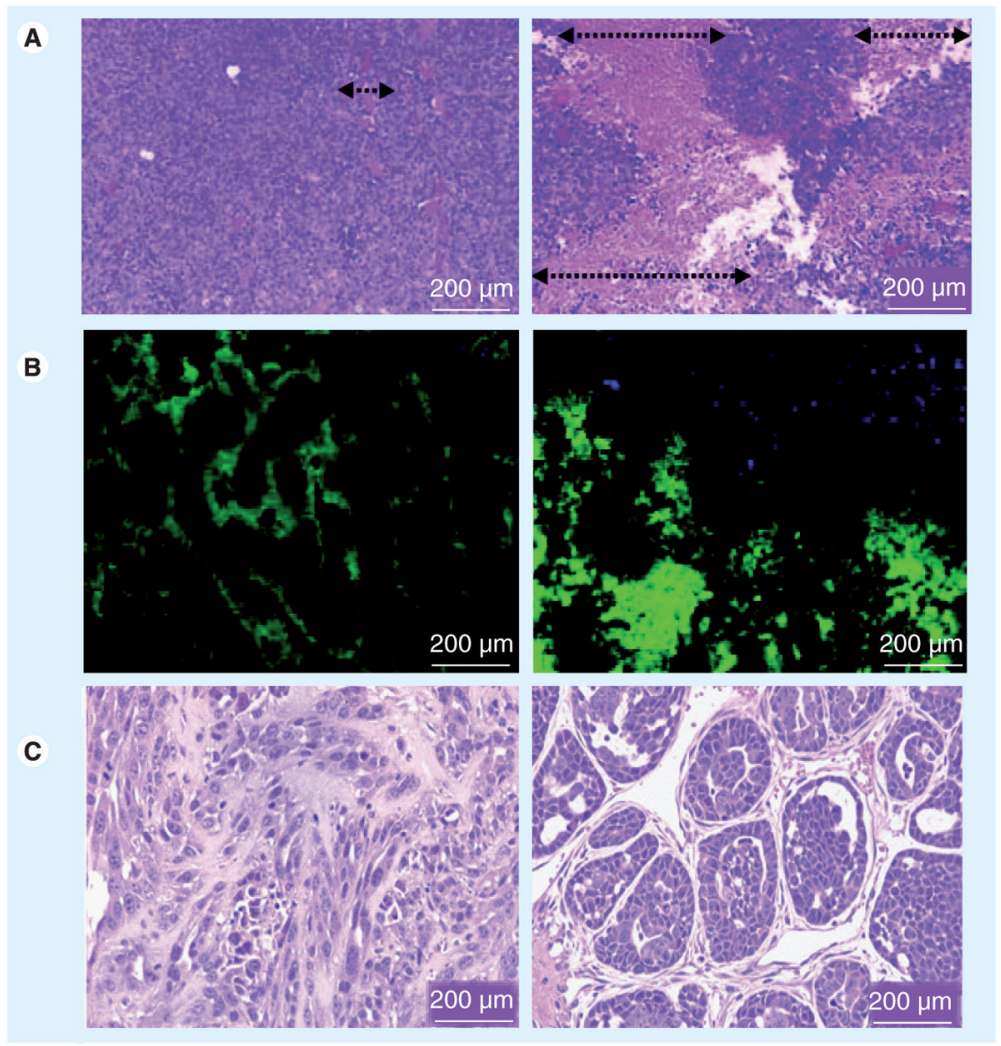

Figure 3. Effects of nanoparticle-mediated hyperthermia on tumors in vivo.

(A) In mice with subcutaneously inoculated human colorectal cancer cells, 90 min following a single 10 Gy dose of radiation therapy using $125 \mathrm{kV}$ x-rays, hematoxylin and eosin-stained slides of the tumor core show minimal necrosis (left), but addition of gold nanoshellmediated hyperthermia $\left(41^{\circ} \mathrm{C}\right.$ for $20 \mathrm{~min}$ immediately prior to radiation) results in significantly more necrosis (right). Arrows denote size of areas of necrosis within tumors [35]. (B) On further investigation, the tumor core of mice treated with radiation alone (left) has classical tissue architecture with central vascular channels surrounded by orderly layers of cells with decreasing levels of oxygenation with increasing distance - hypoxic areas (further from vasculature) are stained green and perfused areas are stained blue in this immunofluorescence image. However, the mice treated with combined hyperthermia and radiation (right) have tumor cores with complete disruption of normal stromal structure, suggestive of vascular collapse [35]. (C) In a mouse model of breast cancer, treatment with a single dose of $6 \mathrm{~Gy}$ with or without post-treatment hyperthermia $\left(42^{\circ} \mathrm{C}\right.$ for $\left.20 \mathrm{~min}\right)$ was followed by tumor digestion $48 \mathrm{~h}$ later and re-implantation in syngeneic mice in limiting dilutions. For reappearance of tumors, the combined treatment group required more cells reimplanted in mice than the radiation alone group, suggestive of a greater effect of combined treatment on putative cancer stem cells. Furthermore, the tumors reappeared as a more aggressive phenotype in the radiation alone group (left) than the combined treatment group (right). These results suggest that more efficient elimination of cancer stem cells by nanoshell-mediated hyperthermia and radiation compared with radiation alone results in lesser ability to recreate tumors as well as appearance of more differentiated, less aggressive and more treatable tumors [46].

Figures reproduced with permission from the referenced sources. 